Pacific Journal of Mathematic 


\section{HARMONIC FUNCTIONALS ON OPEN RIEMANN SURFACES}

\section{Mitsuru Nakai AND Leo Sario}

We denote by $H(R)$ the linear space of harmonic functions on an open Riemann surface $R$ with the topology of uniform convergence on every compact subset. A continuous linear functional on $H(R)$ is referred to as a harmonic functional on $R$; the totality of such functionals is the dual space $H(R)^{*}$ of the locally convex space $H(R)$. A point evaluation $u \rightarrow u(z)$, with $z$ a fixed point of $R$; and a period $u \rightarrow \int_{r} * d u$, with $r$ a fixed cycle on $R$, are the most common examples of harmonic functionals frequently occurring in the theory of functions. We denote by $\left\langle u, h^{*}\right\rangle$ the value of a harmonic functional $h^{*}$ on $R$ at $u$ in $H(R)$. The main purpose of the present study is to establish the following representation of harmonic functionals:

Representation Theorem. Every harmonic functional $h^{*}$ on an open Riemann surface $R$ can be represented by means of a function $h$ harmonic at the point at infinity of $R$ as

$$
\langle u, h *\rangle=\int_{\partial W} u * d h-h * d u
$$

for every $u$ in $H(R)$, where $W$ is any relatively compact subregion of $R$ such that the relative boundary $\partial W$ is smooth and $h$ is harmonic on $R-W$. If $h_{1}$ and $h_{2}$ are functions representing $h^{*}$ in the above sense, then $h_{1}-h_{2}$ can be continued harmonically to all of $R$.

Denote by $H\left(\infty_{R}\right)$ the linear space of germs of functions harmonic at $\infty_{R}$, the point at infinity of $R$ (i.e., the Alexandroff ideal boundary point of $R$ ). The above theorem can be rephrased as the harmonic duality theorem:

$$
H(R)^{*} \cong H\left(\infty_{R}\right) / H(R),
$$

a harmonic version of an algebraic part of the theorem of Köthe [4] and Tillmann [12] (see also Gauthier-Rubel [1]). A closely related treatment of $H(R)^{*}$ when $R$ is a subregion of the Euclidean space $\boldsymbol{R}^{m}$ of dimension $m \geqq 3$ is found in Tillmann [11]; this corresponds to our case of a hyperbolic Riemann surpace $R$. For complete bibliographical information, including a general result of Grothendieck [2], on the Köthe-Tillmann duality, we refer the reader to the monograph of Köthe [5; p. 378]. 
If we denote by $H\left(\infty_{R} ; 0\right)$ the linear space of germs of functions harmonic at $\infty_{R}$ and "vanishing" at $\infty_{R}$, and by $e$ an Evans-SelbergKuramochi potential, which may be viewed as a function harmonic at $\infty_{R}$ with ideal boundary values $e\left(\infty_{R}\right)=+\infty$, then (2) is made precise by the following isomorphisms:

$$
\begin{cases}H(R)^{*} \cong H\left(\infty_{R} ; 0\right) & (R \text { hyperbolic }), \\ H(R)^{*} \cong H\left(\infty_{R} ; 0\right) / \boldsymbol{R}=\boldsymbol{R}[e] & (R \text { parabolic })\end{cases}
$$

Here $\boldsymbol{R}$ is the field of real numbers and $[e]$ is the germ at $\infty_{R}$ which contains $e$. A typical example of a hyperbolic surface is the unit disk $R:|z|<1$; in this case $H\left(\infty_{R} ; 0\right)$ is nothing but the space of harmonic functions on (the neighborhood of) the unit circle $|z|=$ 1 which vanish on $|z|=1$. A point evaluation $u \mapsto u(z)$ is represented, in this case, by the normalized Green function

$$
h(\zeta)=\frac{1}{2 \pi} \log \left|\frac{1-\bar{\zeta} z}{\zeta-z}\right| \in H\left(\infty_{R} ; 0\right) .
$$

A typical example of a parabolic surface is the finite complex plane $R:|z|<+\infty$; here $H\left(\infty_{R} ; 0\right) / \boldsymbol{R}$ is the space of harmonic functions at $\infty_{R}=\infty$ with values zero at $\infty$, and we can take for $e(\zeta)$ the function $\log |\zeta|$. A point evaluation $u \mapsto u(z)$ is represented, in this case, by the normalized Evans function

$$
h(\zeta)=\frac{1}{2 \pi} \log \frac{1}{|\zeta-z|}=-\frac{1}{2 \pi} \log \left|1-\frac{z}{\zeta}\right|-\frac{1}{2 \pi} \log |\zeta|,
$$

where the first summand on the right belongs to $H\left(\infty_{R} ; 0\right) / \boldsymbol{R}$, and the second to $\boldsymbol{R}[e]$. Thus (3) may be interpreted as a generalization of the integral representation of harmonic functions (Poisson's formula).

For the sake of simplicity we restrict our attention to Riemann surfaces, but the entire discussion in the present study applies verbatim also to Riemannian manifolds of any dimension $\geqq 2$. The essence of the proof of the representation theorem lies in the harmonic approximation theorem of Pfluger [7]. We will also give to the Pfluger theorem a proof which is valid not only for Riemann surfaces but also for Riemannian manifolds.

\section{Harmonic approximation.}

1.1. A subregion $\Omega$ of an open Riemann surface $R$ is referred to as a regular subregion if $\Omega$ is relatively compact and the relative boundary $\partial \Omega$ consists of a finite number of disjoint simple analytic closed curves. A normal subregion $\Omega$ of $R$ is a regular subregion 
$\Omega$ of $R$ such that $R-\Omega$ has no compact component. We shall make use of the following "Approximationssatz $H$ " of Pfluger [7, pp. 192-195]:

HARMONIC APPROXIMATION THEOREM. Let $R$ be an arbitrary open Riemann surface, $\Omega$ a normal subregion of $R$, and $F$ a compact subset of $\Omega$. For any harmonic function $u$ on $\Omega$ and any positive number $\varepsilon$, there exists a harmonic function $u_{\xi}$ on $R$ such that $\left|u-u_{\xi}\right|<\varepsilon$ on $F$.

The purpose of the present section is to give a seemingly simpler and shorter proof of this theorem, although it may be less elementary in the sense that we make use of some results outside of function theory proper. Nevertheless, our proof has the merit of wider applicability: we can replace $R$ by any $m$-dimensional $(m \geqq 2)$ noncompact separable connected $C^{\infty}$ manifold, and the harmonicity by the corresponding property defined by any second order linear elliptic partial differential operator invariantly defined on $R$ such that the Dirichlet problem is solvable for every relatively compact subregions of $R$ with a smooth boundary. The proof in this general case is identical with that in the case stated in the above theorem; it will be given in 1.2-1.5.

1.2. We denote by $H(D)$ the linear space of harmonic functions on an open subset $D$ of $R$. For a closed subset $K$ of $R$, we designate by $H(K)$ the linear space of harmonic functions on $K$, i.e., on neighborhoods of $K$. For a regular subregion $\Omega$ of $R$ let $H_{f}^{o}$ be the harmonic function on $\Omega$ with continuous boundary values $f$ on $\partial \Omega$. If $D$ is the union of a finite number of disjoint closed regular subregions and $f$ is a continuous function on $\partial D$, we define $H_{f}^{D}$ in the natural fashion.

To prove the harmonic approximation theorem, we may assume, by enlarging $F$ if necessary, that $F$ is the closure of a normal subregion of $R$. We denote by $C(F)$ the Banach space of continuous functions on $F$ with the supremum norm.

First we prove the theorem for a normal subregion $R$ of a larger open Riemann surface. We set $X=H(R) \mid F=\{h \mid F ; h \in H(R)\}$ and $Y=\left\{H_{f}^{R} ; f \in C(\partial R)\right\} \mid F=\left\{H_{f}^{R} \mid F ; f \in C(\partial R)\right\}$. Since

$$
Y \subset X \subset C(F),
$$

it suffices to prove that $u \mid F$ belongs to $\bar{Y}$, the closure of $Y$ in $C(F)$. Suppose the contrary. Then, by the Hahn-Banach theorem, there exists a $\mu \in C(F)^{*}$, the dual space of the Banach space $C(F)$, 
such that $\langle y, \mu\rangle=0$ for every $y \in Y$ and $\langle u \mid F, \mu\rangle=1$. By the Riesz representation theorem, $\mu$ can be viewed as a signed regular Borel measure on $F$, and the above conditions take the form

$$
\int_{F} H_{f}^{R}(z) d \mu(z)=0
$$

for every $f$ in $C(\partial R)$ and

$$
\int_{F} u(z) d \mu(z)=1
$$

1.3. Take the Green function $g(z, \zeta)$ on $R$ normalized to have the flux 1 across $\partial R$ and consider the Green potential $v(z)=g_{\mu}(z)$ defined by

$$
v(z)=\int_{F} g(z, \zeta) d \mu(\zeta) .
$$

On each side take the normal derivative $\partial / \partial n$ on $\partial R$ toward the interior of $R$, multiply by an arbitrary $f(z)$ continuous on $\partial R$, and integrate along $\partial R$ with respect to the line element $d s$ on $\partial R$. The Fubini theorem and the Poisson type formula yield

$$
\int_{\partial R} f(z) \frac{\partial}{\partial n} v(z) d s=\int_{F} H_{f}^{R}(z) d \mu(z) \text {. }
$$

As a consequence of (4) we have

$$
\int_{\partial R} f(z) \frac{\partial}{\partial n} v(z) d s=0
$$

for every $f$ in $C(\partial R)$, and a fortiori $\partial v / \partial n=0$ on $\partial R$. Clearly the Green potential $v$ has boundary values zero on $\partial R$. Therefore $v$ is the function in $H(R-F)$ with the boundary data

$$
v=\frac{\partial}{\partial n} v=0
$$

on $\partial R$. By the uniqueness of the solution of the Cauchy problem, $v \equiv 0$ on $R-F$, i.e.,

$$
\int_{F} g(z, \zeta) d \mu(\zeta)=0
$$

for every $z$ in $R-F$.

1.4. Let $W \supset F$ be a normal subregion of the region $\Omega$ of the theorem, and denote by $\hat{u}$ the harmonic function on $R-\bar{W}$ with boundary values zero on $\partial R$ and $u$ on $\partial W$. Consider a signed 
measure

$$
d \nu(z)=\frac{\partial}{\partial n}(u(z)-\hat{u}(z)) d s
$$

on $\partial W$, with $\partial / \partial n$ the inner normal derivative on $\partial W$ with respect to the open set $R-\bar{W}$. A simple application of the Green formula gives

$$
u(\zeta)=\int_{\partial W} g(z, \zeta) d \nu(z)
$$

on $\bar{W}$. Since $\partial W$ is contained in $R-F$, we may integrate each side of (6) along $\partial W$ with respect to $d \nu$. By the Fubini theorem and $(7)$,

$$
\int_{\partial F} u(\zeta) d \mu(\zeta)=0
$$

in violation of (5). In view of 1.2 we conclude that $u \mid F \in \overline{H(R) \mid F}$ considered in $C(F)$. This completes the proof in the case of a normal subregion $R$ of a larger open Riemann surface.

1.5. The general case can be deduced from the above special one by a standard approximation method. For the sake of completeness we carry it out. Take a normal exhaustion $\left(R_{n}\right)_{-1 \leqq n<+\infty}$ of $R$, i.e., the $R_{n}$ are normal subregions of $R, \bar{R}_{n-1} \subset R_{n}(n=0,1, \cdots)$, and $R=\bigcup_{-1 \leqq n<+\infty} R_{n}$, such that $\bar{R}_{-1}=F$ and $R_{0}=\Omega$. Set $u_{0}=u$. If $u_{n-1}$ in $H\left(R_{n-1}\right)(n \geqq 1)$ has been chosen, we select $u_{n}$ in $H\left(R_{n}\right)$ such that

$$
\max _{\bar{R}_{n-2}}\left|u_{n-1}-u_{n}\right|<\varepsilon / 2^{n} ;
$$

this is possible in view of the above special case. The sequence $\left(u_{n}\right)_{0 \leqq n \leqq+\infty}$ is uniformly convergent on every compact subset of $R$ to a $u_{\varepsilon}$, say, in $H(R)$. From

$$
\left|u_{0}-u_{n}\right| \leqq \sum_{j=1}^{n}\left|u_{j-1}-u_{j}\right|<\varepsilon
$$

on $F$, we draw the desired conclusion on letting $n \rightarrow+\infty$.

The proof of the harmonic approximation theorem is herewith complete.

1.6. Let $R$ be a noncompact Riemannian manifold, $\Delta$ the Laplace-Beltrami operator on $R, \nabla$ the gradient operator on $R, \boldsymbol{b}$ a $C^{1}$ vector field on $R$, and $c$ a locally Hölder continuous nonpositive function on $R$. The operator 


$$
L u=\Delta u+b \cdot \nabla u+c u
$$

is invariantly defined on $R$. Viewing $H(D)=\left\{u \in C^{2}(D) ; L u=0\right.$ on $D\}$ as the linear space of "harmonic" functions on an open set $D$ of $R$, the entire discussion in 1.1-1.5 remains valid in the present setting. Since the existence of sufficiently many harmonic functions is assured locally, the "harmonic" approximation theorem gives:

BeHnKe-Stein Type Theorem. There exist sufficiently many solutions of the elliptic equation $L u=0$ on $R$.

\section{Spaces of germs.}

2.1. We denote by $\infty=\infty_{R}$ the point at infinity (i.e., the Alexandroff ideal boundary point) of an open Riemann surface $R$. The class $N$ of normal subregions $\Omega$ of $R$ forms a directed set by inclusion, exhausting $R$, i.e., $R=\bigcup_{\Omega \in N} \Omega$. We call a subset $U$ of $R$ containing some $R-\bar{\Omega}(\Omega \in N)$ a neighborhood (actually a punctured neighborhood) of $\infty$. Let $U$ be an open neighborhood of $\infty$, and $h \in H(U)$. We call $h$, or more precisely $(h, U)$, a harmonic function at $\infty$. Let $U_{j}, j=1,2$, be open neighborhoods of $\infty$, and $h_{j}=$ $\left(h_{j}, U_{j}\right)$ harmonic at $\infty$. We say that $h_{1}$ and $h_{2}$ are equivalent if $h_{1} \equiv h_{2}$ on an open neighborhood of $\infty$ contained in $U_{1} \cap U_{2}$. An equivalence class [h] of harmonic functions $h$ at $\infty$ is called a germ of harmonic functions at $\infty$. We denote by $H\left(\infty_{R}\right)$ the set of germs of harmonic functions at $\infty$. By defining addition by $\left[h_{1}\right]+\left[h_{2}\right]=$ $\left[h_{1}+h_{2}\right]$ and scalar multiplication by $\lambda[h]=[\lambda h]$, with $\lambda$ a real constant, we endow $H\left(\infty_{R}\right)$ with the structure of a linear space over the field $\boldsymbol{R}$ of real numbers.

Consider a mapping $h \mapsto[h]$ of $H(R)$ to $H\left(\infty_{R}\right)$. We maintain that it is injective. In fact, if $\left[h_{1}\right]=\left[h_{2}\right]$ for $h_{j} \in H(R)(j=1,2)$, then $h_{1}-h_{2} \in H(R)$ vanishes on a neighborhood $U$ of $\infty$ and therefore on $R$. Thus we may identify the germ [h] containing an $h \in$ $H(R)$ with $h$. In this sense we can view $H(R)$ as a linear subspace of $H\left(\infty_{R}\right)$. We wish to determine the quotient space $H\left(\infty_{R}\right) / H(R)$.

2.2. For $W \in N$, we denote by $N_{W}$ the class of regions $\Omega \in N$ which contain $\bar{W}$. For a function $\psi \in C(\partial W)$ and a region $\Omega \in N_{W}$ we denote by $\psi_{\Omega}$ the function in $C(\partial(\Omega-\bar{W}))$ with $\psi_{\Omega} \mid \partial W=\psi$ and $\psi_{\Omega} \mid \partial \Omega=0$. It is easy to see that the limit

$$
L_{R-\bar{W}} \psi=L_{\psi} \psi=\lim _{\Omega \in N_{W}, \Omega \rightarrow R} H_{\psi_{\Omega}}^{\Omega-\bar{W}}
$$

exists on $R-W$ and the convergence is uniform on each compact 
subset of $R-W$. The mapping $\psi \mapsto L \psi$ defines a linear operator from $C(\partial W)$ into $H(R-W) \cap C(R-W)$ such that $L \psi=\psi$ on $\partial W$ and

$$
\min \left(\min _{\partial W} \psi, 0\right) \leqq L \psi \leqq \max \left(\max _{\partial W} \psi, 0\right)
$$

on $R-W$. Intuitively speaking, $L \psi$ is the harmonic function on $R-\bar{W}$ with boundary values $\psi$ on $\partial W$ and "ideal boundary values zero" at $\infty$. We say that a harmonic function $h=(h, U)$ at $\infty$ vanishes at $\infty, h(\infty)=0$, if $L_{R-\bar{W}} h=h$ on $R-\bar{W}$ for one and hence for every $W \in N$ with $W \supset R-U$. If [h] contains an $h$ with $h(\infty)=0$, then every $u \in[h]$ satisfies $u(\infty)=0$. Such an [h] may be said to vanish at $\infty,[h](\infty)=0$. The class $H\left(\infty_{R} ; 0\right)$ of germs [h] with $[h](\infty)=0$ is a linear subspace of $H\left(\infty_{R}\right)$.

2.3. Observe that $0<L_{R-\bar{W}} 1 \leqq 1$ on $R-\bar{W}$ and either $L_{R-\bar{W}} 1 \equiv$ 1 or $L_{R-\bar{W}} 1<1$ on $R-\bar{W}$. If $L_{R-\bar{W}} 1<1$ on $R-\bar{W}$ for some $W \in N$, then the same is true for every $W \in N$. In this case $R$ is said to be hyperbolic, otherwise parabolic. The parabolicity of $R$ is characterized by the existence of a harmonic function $e(z, \zeta)$ for any $\zeta \in R$, called an Evans-Selberg-Kuramochi potential, on $R-\{\zeta\}$ such that $e(z, \zeta) \rightarrow+\infty$ as $z \rightarrow \infty_{R}$ and $e(z, \zeta)-(1 / 2 \pi) \log |z-\zeta| \rightarrow 0$ as $z \rightarrow \zeta$ for a local parameter $z$ at $\zeta$ (cf. e.g., Sario-Noshiro [10; p. 98], Sario Nakai [9; p. 351]). We shall establish the following decompositions:

$$
\begin{cases}H\left(\infty_{R}\right)=H\left(\infty_{R} ; 0\right)+H(R) & (R \text { hyperbolic }), \\ H\left(\infty_{R}\right)=H\left(\infty_{R} ; 0\right)+H(R)+\boldsymbol{R}[e] & (R \text { parabolic }),\end{cases}
$$

with $e$ an Evans-Selberg-Kuramochi potential. Here

$$
(8)^{\prime}\left\{\begin{array}{rr}
H\left(\infty_{R} ; 0\right) \cap H(R)=\{0\} & (R \text { hyperbolic }), \\
H\left(\infty_{R} ; 0\right) \cap H(R)=\boldsymbol{R}, H(R) \cap \boldsymbol{R}[e]=H\left(\infty_{R} ; 0\right) \cap \boldsymbol{R}[e]=\{0\} & (R \text { parabolic }) .
\end{array}\right.
$$

To prove these relations, take an arbitrary $h=(h, U) \in[h]$ in $H\left(\infty_{R}\right)$. Suppose first that $R$ is hyperbolic. Consider the equation

$$
L(p-h)=p-h,
$$

where $L=L_{R-\bar{\pi}}$ for a $W \in N$ with $W \supset R-U$. It has a unique solution $p \in H(R)$ (cf. Nakai [6]), and $h-p=q \in[q] \in H\left(\infty_{R} ; 0\right)$. This gives the first decomposition $h=q+p$ in (8). If $u \in H\left(\infty_{R} ; 0\right) \cap$ $H(R)$, then $L u=u \in H(R)$, and the maximum principle implies that $u \equiv c$, a constant, on $R$; since $L 1<1, L c=c$ gives $c=0$.

Next suppose $R$ is parabolic. Choose a $W \in N$ with $W \supset R-U$ 
and $\zeta \in W$. Since $\int_{\partial W} * d e(\cdot, \zeta)=1$, we can choose a $\lambda \in \boldsymbol{R}$ with

$$
\int_{\partial W} * d s=0 \quad(s=h-\lambda e(\cdot, \zeta)) .
$$

The parabolicity of $R$ entails

$$
\int_{\partial \Omega} * d L_{R-\bar{w}} \psi=0
$$

for any $\Omega \in N_{W}$ and $\psi \in C(\partial W)$. In fact, by the Green formula, we have

$$
\int_{\partial \Omega}\left(L_{R-\bar{\Omega}} 1\right) * d L_{R-\bar{W}} \psi-\left(L_{R-\bar{W}} \psi\right) * d L_{R-\bar{\Omega}} 1=0,
$$

and $L_{R-\bar{\Omega}} 1 \equiv 1$ gives the above assertion. Therefore $L=L_{R-\bar{W}}$ is a normal operator in the terminology of Rodin-Sario [8], and (9) is a necessary and sufficient condition for the equation

$$
L(p-s)=p-s
$$

to have a solution $p \in H(R)$ (cf. e.g., Rodin-Sario [8; p. 42]). Thus $s-p=q \in[q] \in H\left(\infty_{R} ; 0\right)$ and we obtain the second decomposition $h=q+h+\lambda e$ in (8). Clearly $H\left(\infty_{R} ; 0\right) \cap \boldsymbol{R}[e]=H(R) \cap \boldsymbol{R}[e]=\{0\}$. Let $u \in H\left(\infty_{R} ; 0\right) \cap H(R)$. By the maximum principle, $L u=u \in H(R)$ implies that $u \in \boldsymbol{R}$. This completes the proof of (8) and (8)'.

The decomposition (8) serves to determine the quotient space $H\left(\infty_{R}\right) / H(R)$, which is the main topic of the present section. We repeat here (3) with a view of (2):

$$
\begin{cases}H\left(\infty_{R}\right) / H(R) \cong H\left(\infty_{R} ; 0\right) & (R \text { hyperbolic }), \\ H\left(\infty_{R}\right) / H(R) \cong H\left(\infty_{R} ; 0\right) / \boldsymbol{R}=\boldsymbol{R}[e] & (R \text { parabolic })\end{cases}
$$

3. Harmonic duality.

3.1. Let $C(R)$ be the locally convex space of continuous (not necessarily bounded) functions on $R$ with the topology of uniform convergence on every compact subset of $R$. We shall make use of the following well-known property of $C(R)$ :

The dual space $C(R)^{*}$ of $C(R)$ is the set of signed regular Borel measures $\mu$ on $R$ with compact supports $S_{\mu}$ in $R$.

For the sake of completeness we include a proof. Let $C_{0}(R)$ be the normed space of functions $\psi$ in $C(R)$ with compact supports $S_{\psi}$ in $R$ and with the supremum norm on $R$. The dual space $C_{0}(R)^{*}$ of $C_{0}(R)$ is the set of signed regular Borel measures $\mu$ on $R$ (cf. e.g., Halmos [3; pp. 243-249]). Take an arbitrary $l \in C(R)^{*}$. Its restric- 
tion to $C_{0}(R)$ is continuous with respect to the supremum norm. Thus we can view $l$ as an element of $C_{0}(R)^{*}$, so that

$$
\langle\psi, l\rangle=\int \psi d \mu
$$

for every $\psi \in C_{0}(R)$. Let $\left(\Omega_{n}\right)_{n=1}^{\infty} \subset N$ be such that $\bar{\Omega}_{n} \subset \Omega_{n+1}$ and $R=\bigcup_{n=1}^{\infty} \Omega_{n}$. If $|\mu|\left(R-\bar{\Omega}_{n}\right)>0$ for all $n$, where $|\mu|$ is the total variation of $\mu$, then there exists a $\psi_{n} \in C_{0}(R)$ with support in $R-\bar{\Omega}_{n}$ such that

$$
\int \psi_{n} d \mu \geqq 1
$$

for each $n$. Since $\left(\psi_{n}\right)_{n=1}^{\infty}$ converges to zero uniformly on each compact subset of $R$, the continuity of $l$ on $C(R)$ implies that $\left\langle\psi_{n}, l\right\rangle \rightarrow\langle 0, l\rangle=0$ as $n \rightarrow+\infty$. This is, however, impossible since

$$
\left\langle\psi_{n}, l\right\rangle=\int \psi_{n} d \mu \geqq 1
$$

A fortiori, $S_{\mu}$ is compact. For any $\psi$ in $C(R)$ we can find a sequence $\left(\psi_{n}\right)_{n=1}^{\infty} \subset C_{0}(R)$ converging to $\psi$ uniformly on each compact subset of $R$ and in particular on $S_{\mu}$. Therefore

$$
\langle\psi, l\rangle=\lim _{n \rightarrow \infty}\left\langle\psi_{n}, l\right\rangle=\lim _{n \rightarrow \infty} \int_{S_{\mu}} \psi_{n} d \mu=\int \psi d \mu
$$

for every $\psi$ in $C(R)$, and the assertion on $C(R)^{*}$ follows.

3.2. Since $H(R)$ is a subspace of the locally convex space $C(R)$, any continuous linear functional on $H(R)$ (i.e., $\left.l \in H(R)^{*}\right)$ can be extended to one on $C(R)$ (cf. e.g., Yosida [14; p. 108]). Therefore, $l \in H(R)^{*}$ can be expressed as a signed regular Borel measure $\mu$ on $R$ with compact support $S_{\mu}$ by

$$
\langle u, l\rangle=\int_{S} u d \mu
$$

for every $u$ in $H(R)$. If $H(R)^{\perp}$ is the family of measures $\mu$ in $C(R)^{*}$ such that $\int u d \mu=0$ for every $u \in H(R)$, we have

$$
H(R)^{*} \cong C(R)^{*} / H(R)^{\perp}
$$

For each $\mu \in C(R)^{*}$ the set $\widetilde{S}_{\mu}=\bigcap_{\Omega \in N, \Omega \supset S_{\mu}}$ is compact and $\widetilde{S}_{\mu} \supset S_{\mu}$. Let $\mu \in H(R)^{\perp}$ and $W \in N$ with $W \supset S_{\mu}^{\mu}$. For any $u \in H(W)$ the harmonic approximation theorem guarantees the existence of a sequence $\left(h_{n}\right)_{n=1}^{\infty} \subset H(R)$ such that $h_{n} \rightarrow u$ uniformly on $\widetilde{S}_{\mu}$ as $n \rightarrow \infty$. Hence 


$$
\int_{S_{\mu}} u d \mu=\lim _{n \rightarrow \infty} \int h_{n} d \mu=0
$$

i.e., $\mu \in H(W)^{\perp}$. Let $g_{W}(z, \zeta)$ be the Green function on $W$ and $\zeta \epsilon$ $W-\widetilde{S}_{\mu}$. There exists an $\Omega \in N$ with $\Omega \supset \widetilde{S}_{\mu}$ and $\zeta \notin \Omega$. Since $\mu \epsilon$ $H(\Omega)^{\perp}$ and $g_{W}(\cdot, \zeta) \in H(\Omega)$, we have $\int_{S_{\mu}} g_{W}(z, \zeta) d \mu(z)=0$, i.e., the Green potential

$$
\left(g_{W}\right)_{\mu}=\int g_{W}(\cdot, \zeta) d \mu(\zeta)
$$

vanishes indentically on $W-\widetilde{S}_{\mu}$.

Conversely, let $\mu \in H(R)^{\perp}$ and $W \in N$ with $S_{\mu} \subset W$ and suppose $\left(g_{W}\right)_{\mu} \equiv 0$ on $W-\widetilde{S}_{\mu}$. Let $\Omega \in N$ with $S_{\mu} \subset \Omega \subset \bar{\Omega} \subset W$. For any $h \in H(R)$ we can find, as in 1.4 , a measure $\nu$ with $S_{\nu} \subset \partial \Omega$ such that

$$
h(z)=\int_{S_{\nu}} g_{W}(z, \zeta) d \nu(\zeta)
$$

on $\Omega$. On integrating both sides over $S_{\mu}$ with respect to $d \mu(z)$ and on using the Fubini theorem we obtain

$$
\int_{S_{\mu}} h d \mu=\int_{S_{\nu}}\left(\int_{S_{\mu}} \boldsymbol{g}_{W}(z, \zeta) d \mu(z)\right) d \nu(\zeta)=\int_{S_{\nu}}\left(g_{W}\right)_{\mu}(\zeta) d \nu(\zeta)=0,
$$

i.e., $\mu \in H(R)^{\perp}$. Thus we have the following characterization of $H(R)^{\perp}$ :

$$
\begin{gathered}
H(R)^{\perp}=\left\{\mu \in C(R)^{*} ;\left(g_{W}\right)_{\mu} \equiv 0 \text { on } W-S_{\mu}\right. \\
\text { for any } \left.W \in N \text { with } W \supset S_{\mu}\right\} .
\end{gathered}
$$

We give here an example of a $\mu$ in $H(R)^{\perp}$ which is not zero. Let $\zeta$ be a fixed point in $R$ and $W \in N$ with $\zeta \in W$. Denote by $\delta_{\xi}$ the Dirac measure at $\zeta$ and set

$$
d \mu(z)=d \delta_{\zeta}(z)+\frac{1}{2 \pi} * d g_{W}(z, \zeta),
$$

where the second term on the right is viewed as a measure on $\partial W$. Then $\mu \in C(R)^{*}$ with $S_{\mu}=\{\zeta\} \cup \partial W$ and, for every $f \in C(R)$,

$$
\int_{S_{\mu}} f d \mu=\int_{|\zeta|} f d \delta_{\zeta}+\frac{1}{2 \pi} \int_{\partial W} f(z) * d g_{W}(z, \zeta)=f(\zeta)-H_{f}^{W}(\zeta),
$$

which is zero for $f \in H(R)$, i.e., $\mu \in H(R)^{\perp}$. As this example suggests, the structure of $H(R)^{\perp}$ is quite complicated and therefore the representation (12) does not supply much information on $H(R)^{*}$. To gain more clarity we will try to "sweep" the measure $\mu$ to the ideal boundary of $R$. This is the basic idea we are going to follow 
in the sequel.

3.3. Let $u$ and $h$ be harmonic at $\infty_{R}$ and $W_{j} \in N$ such that $u$ and $h$ are defined on $R-W_{j} \quad(j=1,2)$. Take a $W \in N$ with $\bar{W}_{1} \cup W_{2} \subset W$ and set $\alpha=u * d h-h * d u$. The Green formula gives

$$
\int_{\partial W} \alpha-\int_{\partial W_{j}} \alpha=\int_{W-\bar{W}_{j}}(u d * d h-h d * d u)=0 \quad(i=1,2) .
$$

This means that $\int_{\partial W} \alpha$ is independent of the choice of $W \in N$ such that $u$ and $h$ are harmonic on $R-W$. Therefore we can define

$$
\int_{\partial R} u * d h-h * d u=\lim _{W \in N, W \rightarrow R} \int_{\partial W} u * d h-h * d u,
$$

which can be regarded as a "line integral along the ideal boundary of $R^{\prime \prime}$.

Take an arbitrary $[h] \in H\left(\infty_{R}\right)$ and an $h \in[h]$. The linear functional

$$
u \longmapsto \int_{\partial R} u * d h-h * d u
$$

on $H(R)$ is clearly independent of the choice of $h \in[h]$ (cf. 3.6 below), which we denote by $\tau[h]$ :

$$
\langle u, \tau[h]\rangle=\int_{\partial R} u * d h-h * d u \quad(u \in H(R)) .
$$

The relation $[h] \mapsto \tau[h]$ defines a mapping $\tau: H\left(\infty_{R}\right) \rightarrow H(R)^{*}$, which is clearly linear.

3.4. We maintain that $\tau: H\left(\infty_{R}\right) \rightarrow H(R)^{*}$ is surjective. To see this let $l$ be an arbitrary element in $H(R)^{*}$. We wish to find an $[h] \in H\left(\infty_{R}\right)$ with $\tau[h]=l$. By (11) there exists a signed regular Borel measure $\mu$ on $R$ with compact support $S_{\mu}$ such that

$$
\langle u, l\rangle=\int_{S_{\mu}} u(\zeta) d \mu(\zeta) \quad(u \in H(R)) .
$$

Let $W$ be an arbitrary element of $N$ with $W \supset S_{\mu}$ and denote by $g_{W}(z, \zeta)$ the Green function on $W$ with flux 1 . The Green potential

$$
h_{W}(z)=-\int_{S_{\mu}} g_{W}(z, \zeta) d \mu(\zeta)
$$

is harmonic on $\bar{W}-\widetilde{S}_{\mu}$ and vanishes on $\partial W$. Fix an arbitrary $\Omega \in N$ with $\widetilde{S}_{\mu} \subset \Omega \subset \bar{\Omega} \subset W$. The Green formula and the Fubini theorem yield 


$$
\begin{array}{rl}
\int_{\partial \Omega} u & * d h_{W}-h_{W} * d u=\int_{\partial \Omega} u * d h_{W}-h_{W} * d u \\
& =\int_{\partial W} u * d h_{W}=-\int_{\partial W} u(z) * d_{z} \int_{S_{\mu}} g_{W}(z, \zeta) d \mu(\zeta) \\
& =\int_{S_{\mu}}\left(-\int_{\partial W} u(z) * d g_{W}(z, \zeta)\right) d \mu(\zeta)=\int_{S_{\mu}} u d \mu=\langle u, l\rangle
\end{array}
$$

for every $u \in H(R)$. We have obtained

$$
\langle u, l\rangle=\int_{\partial \Omega} u * d h_{W}-h_{W} * d u \quad(u \in H(R)) .
$$

If $R$ is hyperbolic, then on letting $W \rightarrow R$ in (15) we see that $h_{W}$ converges to

$$
h(z)=-\int_{S_{\mu}} g(z, \zeta) d \mu(\zeta)
$$

uniformly on each compact subset of $R$, where $g(z, \zeta)$ is the Green function on $R$ with flux 1 . From (16) we obtain on letting $W \rightarrow R$ and then $\Omega \rightarrow R$,

$$
\langle u, l\rangle=\int_{\partial R} u * d h-h * d u .
$$

Here $h$ is harmonic at $\infty_{R}$ and the equivalence class $[h] \in H\left(\infty_{R}\right)$ containing $h$ gives the representative $\tau[h]$ which, by (14), is identical with $l$.

If $R$ is parabolic, then there exists a divergent directed net $\left(c_{W}\right)_{W \in N}$ of real numbers such that $\left(g_{W}(z, \zeta)-c_{W}\right)_{W \in N}$ forms a normal family on $R \times R$ less its diagonal set (cf. Tsuji [13; pp. 449-456]). Thus we can choose a sequence $\left(W_{n}\right)_{n=1}^{\infty} \subset N$ which forms an exhaustion of $R$ such that $\left(g_{W_{n}}(z, \zeta)-c_{W_{n}}\right)_{n=1}^{\infty}$ converges to a $\widetilde{g}(z, \zeta)$ uniformly on each compact subset of $R \times R$ less its diagonal set. Using the constants $k_{n}=c_{W_{n}} \mu\left(S_{\mu}\right)$ we set

$$
h_{n}(z)=h_{W_{n}}(z)-k_{n}=-\int_{S_{\mu}} g_{n}(z, \zeta) d \mu(\zeta),
$$

where $g_{n}(z, \zeta)=g_{W_{n}}(z, \zeta)-c_{W_{n}}$. Since $\int_{\partial \Omega} * d u=0$, (16) implies

$$
\langle u, l\rangle=\int_{\partial \Omega} u * d h_{n}-h_{n} * d u \quad(u \in H(R)) .
$$

On letting $n \rightarrow+\infty$ in (17) we see that $h_{n}$ converges to

$$
h(z)=-\int_{S_{\mu}} \widetilde{g}(z, \zeta) d \mu(\zeta)
$$


uniformly on each compact subset of $R$. From (18) we obtain on letting $n \rightarrow+\infty$ and then $\Omega \rightarrow R$

$$
\langle u, l\rangle=\int_{\partial R_{s}} u * d h-h * d u \quad(u \in H(R)) .
$$

As in the hyperbolic case, we also conclude that $\tau[h]=l$.

3.5. We append one more proof of the existence of an $[h] \epsilon$ $H\left(\infty_{R}\right)$ with $\tau[h]=l$ for a given $l \in H(R)^{*}$, valid simultaneously for a hyperbolic and parabolic $R$. We fix a normal exhaustion $\left(W_{n}\right)_{n=1}^{\infty}$ of $R$ and set $v_{n}=h_{W n}$. We start from (16):

$$
\langle u, l\rangle=\int_{\partial \Omega} u * d v_{n}-v_{n} * d u \quad(u \in H(R)),
$$

where $\Omega$ is any element of $N$ with $\widetilde{S}_{\mu} \subset \Omega \subset \bar{\Omega} \subset W_{n}$. Let $w_{\nu}=$ $v_{m+\nu-1}-v_{m+\nu}$ for $\nu=1,2, \cdots$. Clearly $w_{\nu} \in H\left(\bar{W}_{m+\nu}\right)$. By the harmonic approximation theorem, there exists an $s_{\nu} \in H(R)$ such that $\left|w_{\nu}-s_{\nu}\right|<2^{-\nu}$ for $\nu=1,2, \cdots$. The functions

$$
h=v_{m+1}+\sum_{\nu=1}^{\infty}\left(w_{\nu}-s_{\nu}\right)
$$

converge uniformly on each compact subset of $R$. Observe that

$$
h=v_{m+\nu}-\left(s_{1}+\cdots+s_{\nu-1}\right)+\sum_{k=\nu}^{\infty}\left(w_{k}-s_{k}\right)
$$

on $W_{m+\nu}-W_{m}$ for each $\nu=1,2, \cdots$. All three terms on the right are harmonic on $W_{m+\nu}-\bar{W}_{m}$, and the same is true of $h$. Since $\nu$ is arbitrary, we conclude that $h \in H\left(R-\bar{W}_{n}\right)$. In particular, if we set $h=\nu_{m+1}+p$, then $p=\sum_{\nu=1}^{\infty}\left(w_{\nu}-h_{\nu}\right)$ is harmonic on $\bar{W}_{m+1}$ and

$$
\int_{\partial \Omega} u * d h-h * d u=\int_{\partial \Omega} u * d v_{m+1}-v_{m+1} * d u+\int_{\partial \Omega} u * d p-p * d u
$$

for every $u \in H(R)$. The first term on the right is $\langle u, l\rangle$ and, in view of $u, p \in H(\bar{\Omega})$, the second term is zero. Thus

$$
\langle u, l\rangle=\int_{\partial \Omega} u * d h-h * d u \quad(u \in H(R))
$$

and on letting $\Omega \rightarrow R$ we infer that $l=\tau[h]$.

3.6. We denote by $\operatorname{Ker} \tau$ the kernel $\tau^{-1}(0)$ of the linear surjective mapping $\tau: H\left(\infty_{R}\right) \rightarrow H(R)^{*}$. We claim that

$$
\operatorname{Ker} \tau=H(R) \text {. }
$$

If this has been shown, then $H(R)^{*} \cong H\left(\infty_{R}\right) / H(R)$, and the representation theorem ensues. 
To prove (19), take an arbitrary $h \in H(R)$. Then

$$
\langle u, \tau[h]\rangle=\int_{\partial R} u * d h-h * d u=\int_{R}(u d * d h-h d * d u)=0
$$

for every $u \in H(R)$, i.e., $\tau[h]=0$. This means that $\operatorname{Ker} \tau \supset H(R)$. Conversely, let $h=(h, U) \in[h] \in \operatorname{Ker} \tau, W \in N$ with $R-\bar{W} \subset U$, and $\hat{h}_{W}=H_{h}^{W}$. Then from

$$
\left\{\begin{array}{l}
\int_{\partial W} u * d h-h * d u=\int_{\partial R} u * d h-h * d u=\langle u, \tau[h]\rangle=0, \\
\int_{\partial W} u * d \hat{h}_{W}-\hat{h}_{W} * d u=\int_{W}\left(u d * d \hat{h}_{W}-\hat{h}_{W} d * d u\right)=0
\end{array}\right.
$$

for every $u \in H(R)$ it follows on setting $v=h-\hat{h}_{W}$ that

$$
\int_{\partial W} u * d v=0 \quad(u \in H(R)) .
$$

Observe that $v$ vanishes on $\partial W$, is harmonic on $W$ near $\partial W$, and therefore can be extended to a neighborhood $V$ of $\partial W$, i.e., $v \in$ $H(V)$. Since each component of $\partial W$ is conformally a unit circle, $H(\partial W)$ is dense in $C(\partial W)$ on $\partial W$ with respect to the supremum norm. From $\varphi-H_{p}^{W}=0$ on $\partial W$ for any $\varphi \in H(\partial W)$ we see that $H_{\varphi}^{W} \in H(\bar{W})$ for any $\varphi \in H(\partial W)$, and $H(\bar{W})$ is dense in $C(\partial W)$ on $\partial W$ with respect to the supremum norm. The harmonic approximation theorem assures that $H(R)$ is dense in $H(\bar{W})$ on $\bar{W}$ with respect to the supremum norm. Therefore $H(R)$ is dense in $C(\partial W)$ on $\partial W$ with respect to the supremum norm and (20) is valid not only for $u \in H(R)$ but also for $u \in C(\partial W)$. This implies that $* d v=0$ on $\partial W$. In view of $\partial W \subset V, v \in H(V)$, and

$$
v=* d v=0
$$

on $\partial W$, we conclude that $v \equiv 0$ on $V$. Therefore the function $p$ on $R$ defined by

$$
p(z)= \begin{cases}h(z) & (z \in R-\bar{W}), \\ \hat{h}_{W}(z) & (z \in \bar{W})\end{cases}
$$

is harmonic on $R$, and $[h]=[p] \in H(R)$, i.e., $\operatorname{Ker} \tau \subset H(R)$. We have established (19), and the proof of the representation theorem stated in the introduction is complete.

\section{REFERENCES}

1. P. Gauthier and L. Rubel, Holomorphic functionals on open Riemann surfaces, Canad. J. Math., 28 (1976), 885-888.

2. A. Grothendieck, Sur les espaces de solutions d'une classe génerale d'équations aux 
derivées partielles, J. Analyse Math., 2 (1952/53), 243-280.

3. P. Halmos, Measure Theory, Van Nostrand, 1950, $304 \mathrm{pp}$.

4. G. Köthe, Dualität in der Funktionentheorie, J. Reine Angew. Math., 191 (1953), $30-49$.

5. - Topological Vector Spaces I, Springer, 1969, $456 \mathrm{pp}$.

6. M. Nakai, On principal function problem, Nagoya Math. J., 38 (1970), 85-90.

7. A. Pfluger, Theorie der Riemannschen Flächen, Springer, 1957, 248 pp.

8. B. Rodin and L. Sario, Principal Functions, Van Nostrand, 1968, 347 pp.

9. L. Sario and M. Nakai, Classification Theory of Riemann Surfaces, Springer, 1970, $446 \mathrm{pp}$.

10. L. Sario and K. Noshiro, Value Distribution Theory, Van Nostrand, 1966, 236 pp.

11. H. Tillmann, Dualität in der Potentialtheorie, Portugal. Math., 13 (1954), 55-86.

12. - Dualität in der Funktionentheorie auf Riemannschen Flächen, J. Reine Angew. Math., 195 (1956), 76-101.

13. M. Tsuji, Potential Theory in Modern Function Theory, Maruzen, 1959, 590 pp.

14. K. Yoshida, Functional Analysis, Springer, 1965, 458 pp.

Received August 27, 1979. The first author was supported by a Grant-in-Aid for Scientific Research, Japan Ministry of Education, Science and Culture. The second author was supported by Grant MCS 77-16198, National Science Foundation.

Nagoya Institute of Technology

Gokiso, Showa, Nagoya 466

JAPAN

AND

University of California

Los Angeles, CA 90024 



\section{PACIFIC JOURNAL OF MATHEMATICS}

\section{EDITORS}

DONALD BABBITT (Managing Editor)

University of Galifornia

Los Angeles, California 90024

HUgo RossI

University of Utah

Salt Lake City, UT 84112

C. C. MOORE AND ANDREW OGG

University of California

Berkeley, CA 94720

\section{J. DUGUNDJI}

Department of Mathematics University of Southern California Los Angeles, California 90007

R. Finn and J. Milgram

Stanford University

Stanford, California 94305

\section{ASSOCIATE EDITORS}
R. ARENS
E. F. BECKENBACH
B. H. NeUManN
F. WOLF
K. YosHIDA

\section{SUPPORTING INSTITUTIONS}

UNIVERSITY OF ARIZONA

UNIVERSITY OF BRITISH COLUMBIA

CALIFORNIA INSTITUTE OF TECHNOLOGY

UNIVERSITY OF CALIFORNIA

MONTANA STATE UNIVERSITY

UNIVERSITY OF NEVADA, RENO

NEW MEXICO STATE UNIVERSITY

OREGON STATE UNIVERSITY
UNIVERSITY OF OREGON UNIVERSITY OF SOUTHERN CALIFONIA STANFORD UNIVERSITY UNIVERSITY OF HAWAII UNIVERSITY OF TOKYO UNIVERSITY OF UTAH WASHINGTON STATE UNIVERSITY UNIVERSITY OF WASHINGTON 


\section{Pacific Journal of Mathematics}

\section{Vol. 93, No. $1 \quad$ March, 1981}

Richard Arens, Reducing the order of a Lagrangian $\ldots \ldots \ldots \ldots \ldots \ldots \ldots$

Richard Arens, Manifestly dynamic forms in the Cartan-Hamilton treatment

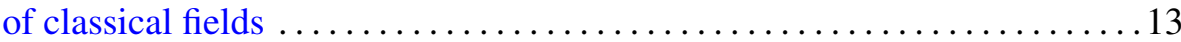

Jimmy T. Arnold, Power series rings over discrete valuation rings $\ldots \ldots \ldots 31$

Charles A. Asmuth and Joe Repka, Supercuspidal components of the

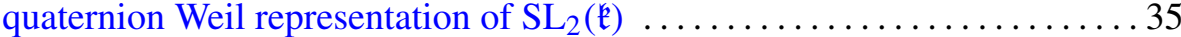

Luis A. Caffarelli and Avner Friedman, Sequential testing of several simple hypotheses for a diffusion process and the corresponding free boundary problem ................................. 49

William B. Jacob, Fans, real valuations, and hereditarily-Pythagorean fields .............................................. 95

W. J. Kim, Asymptotic properties of nonoscillatory solutions of higher order

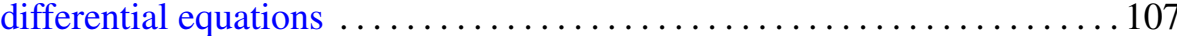

Wayne Steven Lewis, Embeddings of the pseudo-arc in $E^{2} \ldots \ldots \ldots \ldots \ldots 115$

Daniel Alan Marcus, Closed factors of normal Z-semimodules . ......... 121

Mitsuru Nakai and Leo Sario, Harmonic functionals on open Riemann surfaces ............................................. 147

John Currie Quigg, Jr., On the irreducibility of an induced

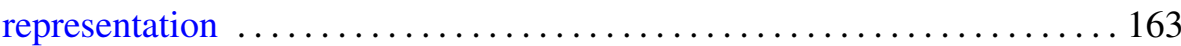

John Henry Reinoehl, Lie algebras and Hopf algebras 181

Joe Repka, Base change for tempered irreducible representations of $\mathrm{GL}(n, \mathbf{R})$

Peter John Rowley, Solubility of finite groups admitting a fixed-point-free automorphism of order $r s t$. I . . . . . . . . . . . . . . . . . . 201

Alan C. Woods, The asymmetric product of three homogeneous linear forms 